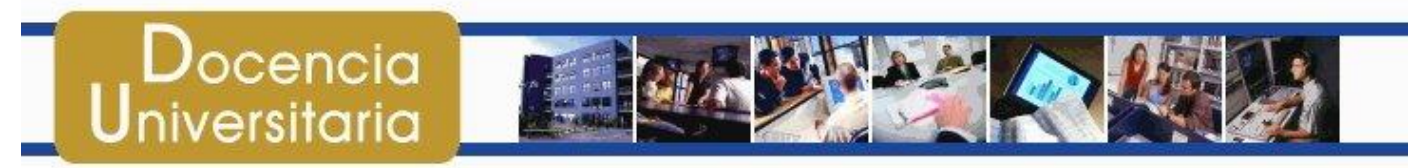

Revista Digital de Investigación en Dacencia Universitaria / Añ̃ 3 - N01- Jun. 2007

\title{
Menos es más \\ Cómo propiciar el aprendizaje autónomo mediante una clase integral en el marco del Modelo Pedagógico UPC
}

\author{
Héctor Ernesto Viale Tudela
}

\section{Resumen}

La exposición presenta el diseño del curso Nivelación de Matemática alineado con el Modelo Educativo de la UPC y desarrollado bajo una estructura que propone el "MATE". En el diseño se considera, a través de una acción intencional, la gestión autónoma del aprendizaje.

Menos es más, la frase que da título a la exposición, encierra un concepto filosófico aplicable al salón de clase que podemos resumir de la siguiente manera: la intervención del profesor debe ser la necesaria y suficiente. La figura del alumno es la que debe destacar. Menos es más busca que el alumno acceda al conocimiento con entusiasmo, lo que ocurrirá si el alumno es el protagonista del sistema de enseñanza-aprendizaje. El profesor debe representar en el aula un recurso más para el aprendizaje del alumno. El conocimiento que debe adquirir un alumno es importante, pero mucho más relevante es el proceso de aprendizaje que logre él mismo. Se espera del profesor que favorezca el aprendizaje de sus alumnos en una atmósfera de tolerancia y respeto. Se le pide además, crear situaciones de aprendizaje variadas y estimulantes en lugar de imponer un conocimiento de forma omnipotente.

Palabras clave: Aprendizaje autónomo, diseño instruccional

\begin{abstract}
'Less is More': How to propitiate Independent Learning in a holistic class through the framework of the pedagogical models of the UPC

This article presents the design of the Math Level 0 course aligned with the Educational Model of the UPC and developed under the structure proposed by 'MATE'.

The phrase 'Less is more' that gives name to this article describes a philosophical concept which is applicable to the classroom where the teacher only intervenes when it is necessary. In this context, the student has the major role.
\end{abstract}




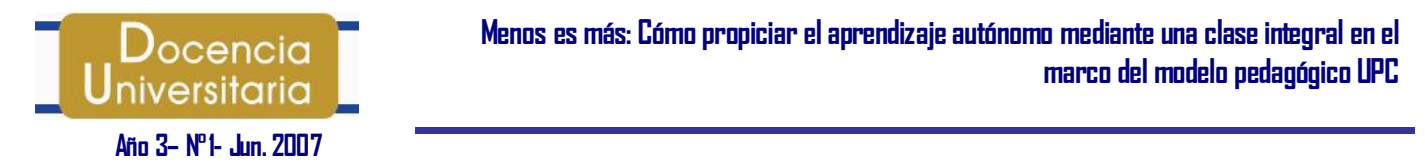

'Less is More' requires the student accessing knowledge with enthusiasm, which will occur if the student has a major role in the teaching-learning system.

The teachers are one more resource in the classroom and while the knowledge that should be acquired is important, the learning process learnt by the student on their own is paramount.

It is expected in 'Less is More' that the teacher favours the learning of the students in an environment of tolerance and respect and it is demanded from the teacher to create a varied and stimulating learning experience instead of imparting knowledge in a patronizing way.

Key words: Independent learning, instructional design. 


\section{Diseño de una clase integral del curso de Nivelación de Matemática}

El Modelo Educativo de la Universidad Peruana de Ciencias Aplicadas (UPC) constituye su filosofía pedagógica, basada en su misión institucional -formar líderes íntegros y elaborar propuestas innovadoras para la creación de una nueva realidad-, y en las competencias generales del estudiante. (Galván 2000: página 4)

La universidad no debe ser únicamente una institución dedicada a la transmisión de conocimientos; si bien es lo primordial, actualmente no es suficiente. "La educación universitaria debe ser para el ser humano, en su calidad de persona, de miembro de la sociedad y de futuro profesional, una experiencia que dure toda la vida en las dimensiones cognitivas, afectivas y prácticas.” (Vélez 2006: página 9)

Tomando lo anterior como premisa, el modelo pedagógico de la UPC sustenta la estructura de sus diseños instructivos en el alumno a través de cuatro fases: motivación, adquisición, transferencia y evaluación, representado por sus siglas como MATE.

El aprendizaje de los niños es principalmente autónomo. En distintas etapas de su crecimiento, el niño va descubriendo y aprendiendo por sí mismo todo aquello que lo rodea. El niño descubre por sí mismo si algo quema, o si algo se rompe al lanzarlo al suelo, o si algo que se lleva a la boca es agradable o no. Por su propia naturaleza, el ser humano, desde sus primeros años de vida, aprende de manera autónoma. ¿En qué momento se pierde esa curiosidad de los niños y su sed natural de aprendizaje? ¿Es la escuela la responsable?

Por otro lado, al crecer y llegar a la adolescencia, se observa cómo nuestros hijos se "fastidian" cuando se les quiere explicar el funcionamiento de cualquier aparato: un equipo de música, un reproductor de discos, etc. Ellos quieren aprender solos y encuentran la mejor forma para lograrlo, tal vez de un modo distinto al de los adultos. En muchas ocasiones, debe reconocerse, lo hacen mejor, pues, como se ve, hay diversas formas de hacer las cosas bien.

Se ha observado cómo los adolescentes no quieren escuchar ni aprender de sus padres. Prefieren hacerlo solos, reafirmando su autonomía. En muchas ocasiones, el costo del aprendizaje autónomo es alto, en la medida en que consideramos que los deben ser.

En el curso de Nivelación de Matemática somos conscientes, desde el primer día de clases, que son ellos los artífices de su futuro inmediato. El alumno es el principal responsable de su propio aprendizaje y nosotros debemos diseñar nuestras clases para asegurar que así sea. Por esto, podemos afirmar que los éxitos de nuestros alumnos son sus éxitos; pero sus fracasos son nuestros fracasos. En la medida en que no logremos 


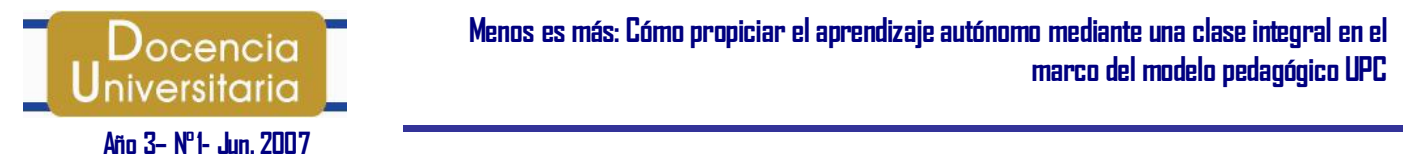

que el alumno aprenda de manera autónoma, seguiremos formando profesionales incapaces de cambiar la sociedad en la que vivimos.

El país necesita profesionales que forjen su futuro y sean los líderes del cambio, capaces de resolver los viejos problemas de la sociedad de una manera creativa.

Nuestras observaciones han sido y son hechas sobre la base de la población más joven de la universidad. Es decir, sobre aquellos alumnos recién ingresados que están convencidos de que alcanzarán el éxito en la universidad siguiendo el sistema de estudio escolar. Este "choque", que es también parte de la formación, puede resultar muchas veces frustrante para el alumno.

Al trabajar con alumnos de este perfil, una de las principales dificultades que hemos encontrado es la paupérrima calidad académica de los alumnos del ciclo inicial. Y con esto no nos referimos al alumno como posibilidad y potencial de crecimiento y desarrollo académico, sino al alumno como resultado de un proceso escolar deficiente.

Hemos encontrado alumnos que, en el proceso de aprendizaje, priorizan la memoria antes que el razonamiento; que anteponen sus temores e inseguridades a la certeza que les pueda brindar una ciencia exacta como la matemática; que no saben priorizar sus actividades ni diseñar un horario de estudios, lo que los hace requerir de más de un ciclo para alcanzar los niveles mínimos que el curso exige para ser promovidos a las asignaturas superiores; que tienen un nivel bajo de concentración y una base escolar muy pobre en Matemática.

Una demostración de este defíciente nivel escolar son los resultados de la prueba diagnóstica (prueba de entrada que evalúa temas de aritmética, álgebra, geometría y trigonometría), que fue aplicada a los alumnos el primer día de clases del semestre 2006-02. El promedio por sección más alto fue de 10,1, mientras el más bajo fue de 05 .

No obstante, más que una dificultad, esto representa un reto para nosotros como docentes. En primer lugar, este reto se traduce en una estrecha coordinación dentro del equipo docente para la aplicación de las estrategias y dinámicas más adecuadas, o para cambiar de rumbo metodológico cuando la situación así lo exija. Esta tarea supera largamente la hora administrativa asignada para tal fin, por lo que la coordinación se extiende a través de celulares y correos electrónicos, los sábados o domingos, en las mañanas o en las noches, o a la hora de almuerzo de los Jueves Culturales, frente a una humeante taza de café. Felizmente, el equipo docente tiene muy claro cuál es su prioridad: el alumno del ciclo inicial. 


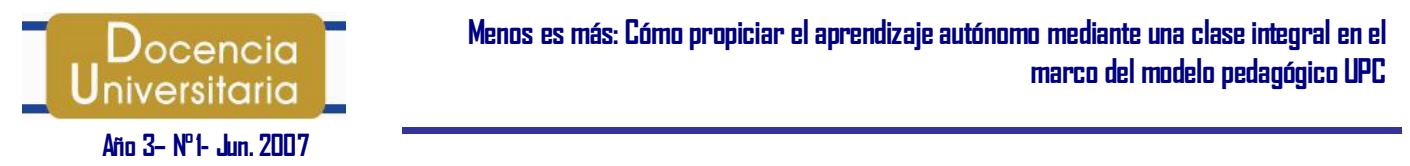

En el semestre pasado fue grande nuestra preocupación al observar los resultados de la primera práctica: el $43 \%$ de los alumnos había aprobado la evaluación, porcentaje mucho menor a los resultados habituales, pues tradicionalmente esta evaluación resultó tener un alto índice de aprobados. Asimismo, nos preocuparon la variabilidad de las calificaciones de una misma sección a lo largo del ciclo académico, y la dispersión de notas entre las secciones ante una misma evaluación.

Como anécdota, recuerdo que en una oportunidad, luego de haber leído en clase la matriz de habilidades de alguna de las evaluaciones, se me acercaron varios alumnos para preguntar qué iba a tomarles en la práctica. Este tipo de preguntas me llevaba a pensar en las grandes y frustrantes limitaciones que existían en la comunicación con mis alumnos. Sin embargo, luego me di cuenta que esta era una característica propia del perfil de nuestros alumnos: inseguridad.

El último día de clases les fue aplicada a los alumnos la misma prueba diagnóstica del primer día de clases, y los resultados fueron notoriamente superiores: la sección que más creció lo hizo en un $147 \%$, y la de menor crecimiento en un $31 \%$.

La importancia de los resultados finales y el progreso académico de los alumnos representa, para nosotros, un aliciente para seguir trabajando en este difícil escalón de la vida universitaria por el cual debe transitar un alto porcentaje de los alumnos. Nuestro objetivo es guiarlos hacia un aprendizaje basado en el entendimiento, la reflexión y el análisis serio.

Por ello, debemos apuntar a que el alumno encuentre el camino: que plantee las preguntas y alcance las respuestas. Nuestras respuestas deben estar planteadas como preguntas para ayudar al alumno a alcanzar su propia conclusión. Es imprescindible que el alumno cuestione y sea crítico.

La tecnología facilita el aprendizaje autónomo. Un buen diseño de actividades de aprendizaje autónomo basado en el uso de las herramientas de la tecnología y la información contribuye a que el alumno desarrolle su autonomía. Resulta fundamental el papel que desempeña el alumno en su proceso de aprendizaje y el respectivo uso de los recursos educativos puestos a su disposición en el Aula Virtuall.

El diseño del curso Nivelación de Matemática está alineado con el Modelo Educativo de la UPC. Cuenta con los esquemas pedagógicos estructurados -como se señaló anteriormente-, siguiendo las fases de motivación, adquisición, transferencia y

\footnotetext{
${ }^{1}$ Cfr. Manrique 2004: página 3
} 


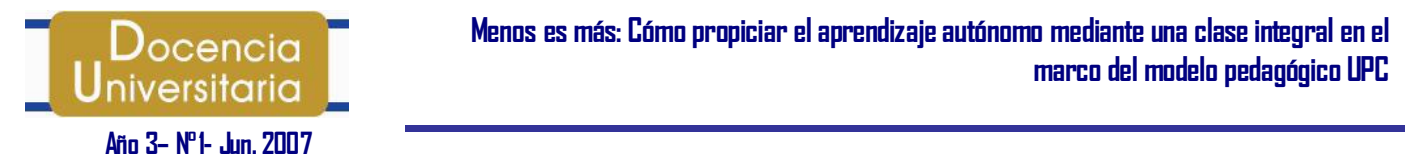

evaluación. Del mismo modo, cuenta con matrices de evaluaciones, criterios de calificación, clases integrales, proyectos integradores, etc. Desde el mismo diseño del curso, debe considerarse, a través de una acción intencional, la gestión autónoma del aprendizaje2.

En la experiencia de promover el aprendizaje autónomo, hemos escuchado opiniones muy interesantes de los alumnos: "Nunca había trabajado tanto en una clase de matemática", "Si así hubiese sido en el colegio, Matemática hubiera sido mi curso preferido", "Hoy mis neuronas han sudado", entre otras.

Menos es más, la frase que da título al presente ensayo, encierra un concepto filosófico aplicable al salón de clase que podemos resumir de la siguiente manera: la intervención del profesor debe ser la necesaria y suficiente. La figura del alumno es la que debe destacar. Menos es más busca que el alumno acceda al conocimiento con entusiasmo, lo que ocurrirá si el alumno es el protagonista del sistema de enseñanza-aprendizaje. El profesor debe representar en el aula un recurso más para el aprendizaje del alumno. El conocimiento que debe adquirir un alumno es importante, pero mucho más relevante es el proceso de aprendizaje que logre él mismo. Se espera del profesor que favorezca el aprendizaje de sus alumnos en una atmósfera de tolerancia y respeto. Se le pide además, crear situaciones de aprendizaje variadas y estimulantes en lugar de imponer un conocimiento de forma omnipotente.

Menos es más pretende colocar en el centro del sistema de enseñanza-aprendizaje al alumno, alrededor del cual debe girar la institución, el profesor y los conocimientos. Menos es más también busca minimizar el protagonismo del profesor en el salón de clases.

Existen diversas actividades que pueden conducir al aprendizaje autónomo del alumno: por ejemplo, pedirles que lean la matriz de habilidades en voz alta y que expliquen lo que han comprendido, o que brinden ejemplos o contextualicen alguna situación real.

En muchas ocasiones, los alumnos no tienen confianza en sus propias capacidades. En estos casos, lo que necesitan es que alguien confíe en ellos. Esta confianza se les da cuando se empieza a contar con ellos como parte fundamental en el desarrollo de la clase. Menos es más confía en el alumno y rescata de él la confianza y la seguridad necesarias. Gracias a esto, los alumnos logran una gran confianza en sí mismos, en sus competencias y en su potencial de aprendizaje: no se sienten inferiores a nadie, y se convencen de que es posible salir adelante. Menos es más está alineado con la filosofía

${ }^{2}$ Cfr. Manrique 2004: página 7 


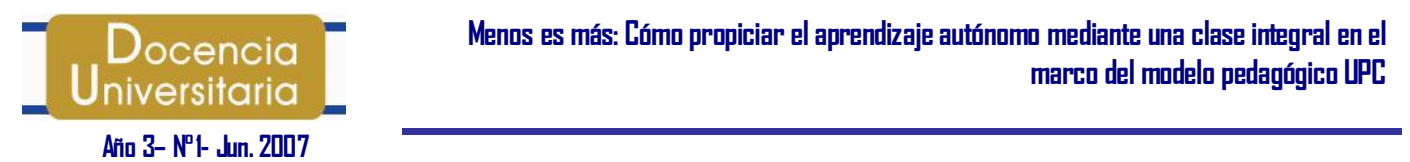

de Esa Räty, director del colegio Niinivaara (Joensuu, Finlandia), que postula que "cada alumno es importante".

En la línea de Menos es más, fueron tres los profesores del área de Ciencias que participaron directamente. Por un lado, Manolo Álvarez y Alberto Mejía diseñaron la metodología del curso Introducción a la Matemática Básica y, por otro, Yuri Milachay diseñó la metodología del curso Introducción a la Física Básica, ambos cursos pertenecientes al Taller de Selección Preferente (SP), que está dirigido a los alumnos que pertenecieron al tercio superior de sus respectivos colegios. El objetivo fue incentivar el aprendizaje autónomo del alumno, apoyado por un importante componente virtual y por el uso de las TICs (Tecnologías de la Información y Comunicación). Los alumnos de este taller no tuvieron la obligación de estar matriculados en él ni de entregar o enviar los trabajos a través del Aula Virtual. La calificación obtenida por ellos no tuvo ninguna relevancia en los cursos posteriores de su carrera. Sin embargo, se logró que el alumno se comprometiera con su propia educación, volviéndose el principal artífice de su formación académica.

Voy a reseñar ahora algunas anécdotas producidas en la aplicación de Menos es más. El ciclo pasado, una alumna que estaba repitiendo el curso de Nivelación de Matemática me dijo que no tenía la más mínima idea de cómo plantear y resolver problemas. Tampoco sabía cómo definir las variables que luego la conducirían a plantear las ecuaciones, ni cómo dar la respuesta correcta como resultado del análisis o discriminación del caso. Por ello, me pedía que resolviera varios problemas en la pizarra para que ella y sus compañeros pudiesen aprender. Yo le respondí que ella estaba en serios aprietos, pues de ninguna manera yo resolvería los problemas en la pizarra, ya que lo único que conseguiría con esto sería que los alumnos copiaran mi forma de pensar. Menos es más busca que los alumnos sean autónomos y auténticos y que lleguen a los resultados por sus propios caminos. El verdadero problema al cual esta alumna se enfrentaba era la elaboración de una estrategia para resolver el problema. La alumna pasó el curso y luego me escribió, en la última tarea del curso, unas líneas de agradecimiento.

Otra anécdota de Menos es más es el caso de otra alumna que estaba repitiendo el mismo curso, y en las evaluaciones presentaba todos los problemas resueltos de una forma muy original, con diagramas y gráficos muy claros. Cuando le pregunté por qué dibujaba esos gráficos en las evaluaciones, me contestó que así entendía mejor el problema y la ayudaba a pensar. La alumna pasó el curso con una alta calificación y en la última tarea del curso escribió unas líneas en las que decía que me amaba. La filosofía de Menos es más, sin proponérselo, establece vínculos afectivos con los 


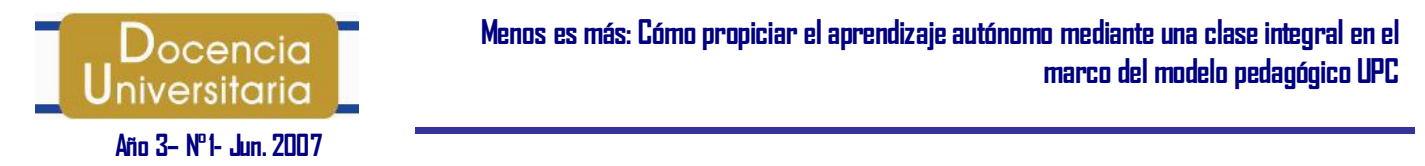

alumnos, permitiendo que estos generen sus propias estrategias, no solo de resolución de problemas, sino de aprendizaje.

Asimismo, la metodología propicia un ambiente distendido, sin que ello implique muestras de indisciplina o irrespeto. Muchas veces basta con una mirada fija para evitar cualquier amago de distracción individual o colectiva dentro del aula.

Podría decirse que Menos es más no es únicamente una metodología que promueve el aprendizaje autónomo; Menos es más es un estilo de vida.

En el anexo 2, podemos observar los resultados obtenidos en las secciones del curso Nivelación de Matemática en las que se aplicó la filosofía del Menos es más.

A continuación les mostramos una experiencia del curso Nivelación de Matemática de la UPC. El diseño de esta clase se llevó a cabo en la Clase Integral del curso.

\section{Relato de la Experiencia}

En el marco del concepto de Menos es más, el uso de la tecnología facilita la aplicación del aprendizaje autónomo y así lo hemos entendido en el curso Nivelación de Matemática. De esta manera, hemos diseñado las clases de manera tal que el protagonista de estas sea el alumno y no el profesor.

La aplicación de este concepto exige un diseño de la clase que debe ser debidamente estudiado y revisado. La participación del profesor se concentra en la etapa previa al inicio de las clases: en la etapa del diseño, mas no durante el desarrollo de la clase.

La Clase Integral obedece al diseño de una clase compartida con el tutor de la sección y se lleva a cabo durante la última sesión de la semana. Diseñada principalmente sobre la base de dinámicas individuales y grupales, la Clase Integral busca desarrollar la autonomía del estudiante. Tiene una duración de dos horas, cerrando la semana. El diseño contempla la aplicación de una breve evaluación (que llamaremos "Control") que, tras ser corregida, sirve para retroalimentar inmediata e individualmente a cada uno de los alumnos.

Al inicio de la Clase Integral, el profesor del curso y el tutor agrupan a los alumnos siguiendo un patrón determinado: se puede optar por conformar grupos homogéneos, heterogéneos o aleatorios. Esto debe definirse en alguna de las reuniones de coordinación del curso, de modo que se trabaje por igual en todas las secciones. La experiencia nos dice que es preferible agrupar a los alumnos en grupos interiormente 


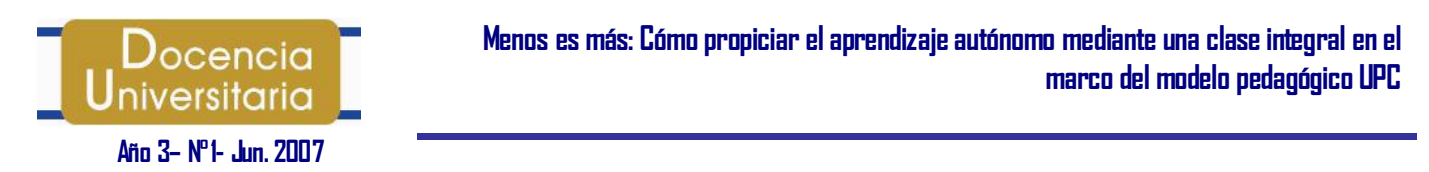

heterogéneos, pero exteriormente homogéneos3. De esta manera tendremos en cada grupo a alumnos con perfiles académicos muy diferentes, lo cual contribuirá al apoyo entre todos.

A los alumnos se les explica que esta clase integral tiene cinco4 etapas y que la nota final se obtiene de la suma de los puntajes conseguidos en cada etapa. Los problemas y/o modelaciones del "Control" son contextualizados (en lo posible, con la realidad) y diseñados de manera tal que puedan integrar los conocimientos $\mathrm{y}$ habilidades matemáticas, desarrollados hasta ese momento. (Motivación)

Luego, cada alumno recibe dos hojas del "Control". En una de ellas, los alumnos resuelven de forma individual las preguntas planteadas. La filosofía del Menos es más indica que en esta etapa es importante advertir a los alumnos que ni el profesor ni el tutor van a asesorarlos durante el desarrollo de la Clase Integral; esta debe ser un espacio de trabajo exclusivo del alumno. Si este tiene alguna duda, le está permitido consultar su cuaderno o el manual del curso. (Adquisición - Transferencia)

Si bien en esta etapa el trabajo es personal, es importante que los alumnos ya se encuentren agrupados, debido al efecto psicológico "de equipo" que se genera. Muchas veces, en el mundo laboral nuestras actividades son individuales, pero no dejamos de ser conscientes de que esta labor individual repercute también en nuestros compañeros de trabajo.

Para la aplicación del "Control" se estima una duración de 30 minutos, luego de los cuales el profesor indicará a los alumnos que escriban únicamente sus respuestas (no así el desarrollo) en la segunda hoja. Posteriormente, el profesor recogerá las hojas con el desarrollo realizado por cada alumno. La nota obtenida por el alumno equivaldrá al $60 \% 5$ de la nota de la Clase Integral. (Evaluación)

Luego, el profesor indicará a los alumnos que tienen 20 minutos para discutir las respuestas al interior de cada grupo (no se pueden hacer consultas con los alumnos de los otros grupos). Se designa, de manera rotativa, un moderador por cada grupo. En el caso de que existan respuestas diferentes, cada alumno debe defender su punto de vista hasta que alguno de ellos persuada al resto de que su respuesta es la correcta. Para lograrlo, debe fundamentar correctamente su punto de vista. Estas discusiones deben ser supervisadas por el profesor y el tutor, pero por ningún motivo ellos pueden intervenir

\footnotetext{
${ }^{3}$ Pensamos que la distribución de los alumnos en las aulas de los cursos masivos debe hacerse bajo el mismo criterio. De esta manera se evita tener una alta dispersión de las notas entre las distintas secciones. ${ }^{4}$ Depende del diseño de la Clase Integral.

${ }^{5}$ Este porcentaje puede variar según el diseño de la clase.
} 


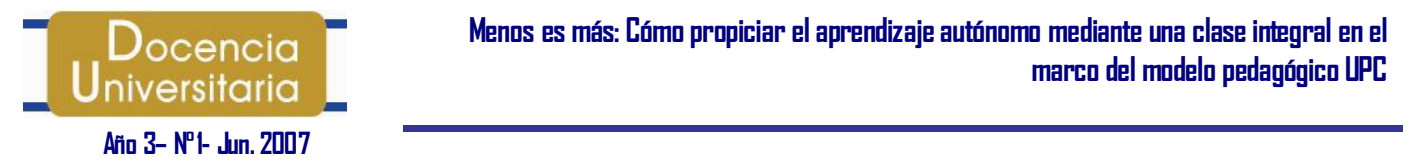

ayudando a los alumnos. En todo caso, el profesor y el tutor pueden responderle al alumno con una pregunta. (Evaluación)

Si en esta etapa algún alumno, convencido de que su respuesta no es la correcta, tiene que cambiarla, debe hacerlo en la segunda hoja después de la respuesta obtenida por él en la etapa anterior. En esta segunda hoja no debe realizarse ningún cálculo. Solo se escriben las respuestas.

Hasta ese momento, los alumnos no conocen las respuestas correctas a los problemas planteados en el "Control". Lo único que poseen es un acuerdo sobre la posible respuesta a las preguntas. Los alumnos de los otros grupos podrían tener una respuesta distinta para la misma pregunta.

Tras 20 minutos de discusión, los alumnos se reagrupan de manera tal que cada nuevo grupo tenga a un alumno de cada grupo anterior. En esta etapa se les brinda 15 minutos para que intercambien ideas y argumenten a favor o en contra de las respuestas obtenidas en la discusión grupal previa. Los alumnos deben registrar nuevamente en la segunda hoja los cambios ocurridos en sus respuestas. Hasta entonces, los alumnos siguen sin conocer las respuestas correctas a las preguntas del "Control".

Inmediatamente después, los alumnos regresan a su grupo original y se les da 10 minutos para preparar el documento final con las respuestas obtenidas luego de las dos discusiones grupales. El documento final es una hoja del "Control" en la que deben anotar el nombre de todos los integrantes del grupo original, y señalar únicamente las respuestas a cada una de las preguntas.

Finalmente, se resuelve el control en la pizarra. Para este fin, el profesor invita a un alumno de cada grupo para resolver los problemas (el alumno es elegido al azar). Esta actividad debe realizarse en la pizarra con cada alumno por separado. El profesor evalúa la exposición de los alumnos: el uso de pizarra, la claridad de los conceptos, la corrección del vocabulario, la elaboración de alternativas de solución, etc.

Tanto los alumnos como el profesor y el tutor pueden plantearle preguntas al alumno expositor. A modo de cierre de cada pregunta resuelta en la pizarra, el profesor interviene para añadir algún comentario refiriéndose a situaciones contextualizadas, similares a las presentadas en el problema integrador.

Es recién en esta etapa en la que el alumno se entera de las respuestas a las preguntas del "Control". (Retroalimentación) 


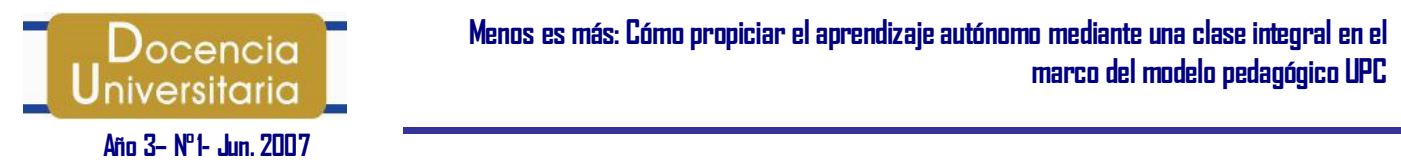

La nota asignada al alumno expositor será la nota del grupo. Esta nota representará el $20 \%$ de la calificación total de la Clase Integral. El 20\% restante será obtenido de la nota asignada por el correcto desarrollo de las tareas dejadas para la casa durante la semana. 


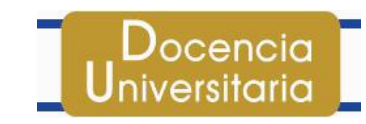

Año 3- No'- Jun. 2007

\section{Anexo 1}

Ludwig Mies van der Rohe (Aquisgrán, Alemania, 27 de marzo de 1886 - , 17 de agosto de 1969) fue un arquitecto y diseñador alemán. Nació como el más joven de los cuatro hijos de una familia católica propietaria de un negocio de escultura y un taller de cantería. Tras terminar sus estudios en la Escuela de Artes y Oficios, empezó a trabajar en el taller de su padre, donde adquirió un gran conocimiento y dominio en el trabajo de la piedra.

Posteriormente se incorporó al despacho de arquitectura de Bruno Paul en Berlín. Mientras trabajaba en este despacho, recibió su primer encargo como arquitecto: la casa del catedrático de Filosofía de la Universidad de Berlín: Alois Riehl. Este proyecto le abrió el acceso a los círculos de la sociedad de los que procederían sus futuros clientes, sobre todo intelectuales y artistas, así como hombres de negocios de la industria y las finanzas.

Unos años después trabajó en el despacho de Peter Behrens, bajo cuya influencia Mies desarrolló un estilo arquitectónico basado en técnicas estructurales avanzadas y en el clasicismo prusiano. También realizó diseños innovadores con acero y vidrio. En aquellos años, Mies colaboró con la revista "G" e hizo contribuciones importantes a la filosofía de la arquitectura como director del proyecto Weissenhof, el cual tenía por objetivo investigar innovaciones en los prototipos de vivienda moderna, $\mathrm{y}$, sobre todo, como director de la famosa Escuela de la Bauhaus.

Mies decidió abandonar Alemania a fines de 1930 cuando vio cómo el nazismo se iba volviendo cada vez más poderoso. Se marchó a los Estados Unidos, país donde ya era conocido como un arquitecto y diseñador influyente. En Chicago fue nombrado Decano de la Escuela de Arquitectura del Instituto Tecnológico Armour (en la actualidad Instituto Tecnológico de Illinois). Su única condición para aceptar el puesto fue de poder rediseñar los edifícios del recinto universitario. Todavía hoy siguen en pie algunos de ellos, incluyendo la sede central del Colegio de Arquitectura.

En 1958, Mies construyó el edificio Seagram en Nueva York, el cual fue considerado como la máxima expresión de la arquitectura internacional. Se trata de un edifico de fachadas acristaladas, de líneas claras y sobrias, construido según la técnica del "muro cortina" desarrollada por Mies, que consiste en un fachada sin función de sustentación, la cual recae en pilares situados detrás de esta. Mies recibió numerosos encargos para diseñar edificios de todo tipo, incluidos algunos rascacielos que siguen siendo totalmente actuales en su diseño a pesar de los años transcurridos. A lo largo de su vida profesional, Mies luchó por conseguir una arquitectura de carácter universal y simple, y que fuese honesta en el empleo de los materiales y en las estructuras. Fue así como hizo 


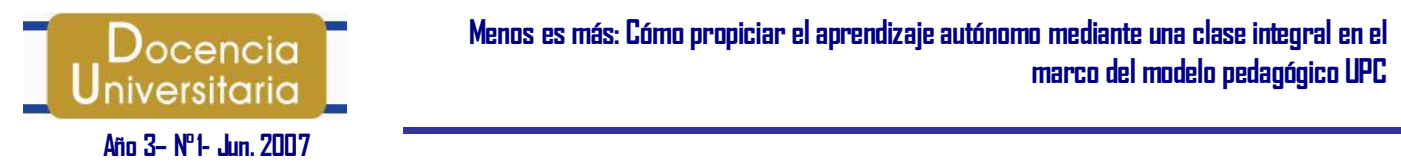

célebre la frase "Less is more" ("Menos es más"), la cual se convirtió en el lema de la arquitectura de vanguardia de la primera mitad del Siglo XX y, al mismo tiempo, en uno de los conceptos más discutidos en la teoría arquitectónica.

Tomado de: Ludwig Mies van der Rohe. Wikipedia, la enciclopedia libre 


\section{Anexo 2}

Porcentaje de alumnos aprobados por semestre por sección

\begin{tabular}{|c|c|c|c|}
\hline Semestre & $\begin{array}{c}\text { \% Promedio de } \\
\text { alumnos aprobados }\end{array}$ & Sección & $\begin{array}{c}\text { \% De alumnos } \\
\text { aprobados }\end{array}$ \\
\hline $2004-01$ & 48,6 & Z012 & 45,42 \\
\hline $2004-02$ & 31,2 & Z004 & 48,35 \\
\hline $2005-00$ & 72,4 & Z001 & 48,15 \\
\hline $2005-01^{7}$ & 46,7 & Z005 & 60,14 \\
\hline $2005-02$ & 46,7 & Z017 & 61,31 \\
\hline & & Z003 & 51,50 \\
\hline $2006-01$ & 40,6 & Z006 & 51,30 \\
\hline & & Z006 & 63,77 \\
\hline $2006-02$ & 51,4 & Z007 & 52,42 \\
\hline
\end{tabular}

\footnotetext{
${ }^{6}$ De todas las secciones.

${ }^{7}$ A partir del semestre 2005 - 01 se empezó a aplicar la filosofía del Menos es más en las secciones indicadas.
} 
Año 3- No'- Jun. 2007

\section{Bibliogra fía}

GALVÁN, Liliana y GOLEGANT, Jonathan

2006 Técnicas de Enseñanza-Aprendizaje. Lima: UPC

MANRIQUE, Lileya

2004 El Aprendizaje Autónomo en la Educación a Distancia. Lima: Pontificia Universidad Católica del Perú

ROBERT, Paul

2006 La Educación en Finlandia: Los secretos de un éxito asombroso. Traducción de Manuel Valdivia Rodríguez (Perú) http://blog.pucp.edu.pe/media/229/20070219-

07\%20Finlandia\%20y\%20PISA\%202.doc)

VÉLEZ, Odette

2006 Modelo Educativo de la UPC

En: Buenas Prácticas Universitarias en Materia de Calidad. Lima: UPC

Para citar este documento, puede utilizar la siguiente referencia:

VIALE, Héctor (2007). "Menos es más: Cómo propiciar el aprendizaje autónomo mediante una clase integral en el marco del Modelo Pedagógico UPC" [artículo en línea]. Revista Digital de Investigación en Docencia Universitaria (RIDU) Año 3 -No1-Junio 2007. [Fecha de consulta: dd/mm/aa]. <

http://beta.upc.edu.pe/calidadeducativa/ridu/2007/ridu3 2HV.pdf $>>$

\section{Héctor Ernesto Viale Tudela}

hviale@upc.edu.pe

Ingeniero Civil, egresado de la maestría en Gestión de la Educación de la Pontificia Universidad Católica del Perú. Profesor de los cursos de Nivelación de Matemática, Matemática Básica y Cálculo del Área de Ciencias, UPC, Coordinador del curso de Nivelación de Matemática y Coordinador del Ciclo Inicial, UPC. Ganador del Premio al Modelo Pedagógico UPC (2006) otorgado por el Departamento de Calidad Educativa. Actualmente se encuentra cursando el Diplomado de Docencia Universitaria en la Universidad Peruana de Ciencias Aplicadas. 